\title{
Preparación para una emergencia: el análisis de la pandemia covid-19 en Argentina, Brasil y México
}

\author{
Diego Silva Jiménez \\ diego.silva@ucentral.cl \\ https://orcid.org/0000-0003-2818-211X \\ Administrador Público \\ Magíster en Gerencia y Políticas Públicas. \\ Académico de la Unidad de Salud Pública \\ del Departamento de Formación Transversal en Salud, \\ Facultad de Ciencias de la Salud, de la Universidad Central de Chile, \\ Santiago, Chile \\ Romina Lizondo Valencia \\ rlizondov@ucentral.cl \\ https://orcid.org/0000-0003-1085-9524 \\ Enfermera, Magister en Gestión Educacional y \\ Magister en educación con mención en investigación, \\ Académica Carrera de Enfermería Universidad Central de Chile, \\ Santiago, Chile
}

\author{
Diego Arancibia Morales \\ diego.arancibia@ucentral.cl \\ https://orcid.org/0000-0002-0079-6809 \\ Cientista Político, Magíster en Seguridad y Defensa. \\ Académico del Instituto de Investigación y Postgrado, \\ Facultad de Ciencias de la Salud, Universidad Central de Chile, \\ Santiago, Chile \\ Paula Beatriz Méndez Celis \\ paula.mendez@ucentral.cl \\ https://orcid.org/0000-0002-1335-0734 \\ Enfermera, Magister en Docencia de Educación Superior, \\ Doctora en Enfermería. Académica Facultad de Enfermería \\ Universidad Andrés Bello, Santiago, Chile,
}

\section{RESUMEN}

Durante el año 2020 el covid-19 azotó a la humanidad, lo que llevó a los países a activar protocolos de preparación de emergencias, con distintas acciones y resultados. El objetivo de esta investigación fue analizar la preparación para la emergencia del Covid-19 en Argentina, Brasil y México. La metodología utilizada fue cualitativa, descriptiva de análisis documental sobre estos países. Para el análisis se utilizó el documento Lista de 
verificación para gestionar los riesgos y los efectos de COVID-19 de la Organización Panamericana de la Salud a través del enfoque preparación para una emergencia. Como conclusión, los países analizados contaban con cierto nivel de preparación para afrontar la pandemia, así como una coordinación en términos jurídicos sólida, sin embargo, en la acción se vio el fracaso de la comunicación de riesgo y la participación comunitaria, ejes fundamentales para haber afrontado de mejor manera esta crisis.

Palabras clave: análisis cualitativo; comunicación; emergencia; pandemia; salud pública 


\title{
Preparing for an emergency: the analysis of the covid-19 pandemic in Argentina, Brazil and Mexico
}

\begin{abstract}
During 2020, covid-19 hit humanity, making the countries to activate emergency preparedness protocols, with different actions and results. The objective of this research was to analyze the preparedness for the Covid-19 emergency in Argentina, Brazil and Mexico. The methodology used was qualitative, descriptive of documentary analysis on these countries. For the analysis was used the document Checklist for managing the risks and effects of COVID-19 of the Pan American Health Organization through the approach of preparing for an emergency. In conclusion, the countries analyzed had a certain level of preparedness to face the pandemic, as well as coordination in solid legal terms, however, the action saw the failure of risk communication and community participation, fundamental axes to have dealt with this crisis in a better way.
\end{abstract}

Keywords: qualitative analysis; communication; emergency; pandemic; public health

Artículo recibido: 02 Setiembre. 2021 Aceptado para publicación: 30 Setiembre. 2021 Correspondencia: paula.mendezce@gmail.com Conflictos de Interés: Ninguna que declarar 


\section{INTRODUCCIÓN}

El Covid -19 ha desatado una pandemia a nivel mundial "generando colapso del sistema sanitario en muchas regiones del planeta, por ser muy contagioso, causando miles de muertes especialmente en adultos mayores con comorbilidades como diabetes mellitus o hipertensión arterial.” (Maguiña, Gastelo \& Tequen, 2020, p. 129).

Bajo este contexto el Covid-19 vino a demostrar que las amenazas a la seguridad de los Estados no implican solo cuestiones de protección a la soberanía nacional y su integridad territorial, sino que incluye otros riesgos y amenazas, entre los cuales se encuentran las pandemias. Ante esto cabe preguntarse si los países consideraron los efectos de una pandemia desde la perspectiva político-estratégica, asociado a los efectos negativos sobre bienestar socio económico de un país; efecto que se ven amplificados por factores como la globalización, el cambio climático, los niveles de urbanización, procesos migratorios y turismo, o incluso las condiciones socioeconómicas locales de los diferentes países, entre otros. (Rodríguez, et al. 2020)

El primer acercamiento sobre como una enfermedad podría afectar la seguridad de los Estados se desarrolló en Ciudad de México, en la Conferencia Especial sobre Seguridad, de la Organización de Estados Americanos (OEA), donde establecen que dicha seguridad se puede ver afectada por amenazas de naturaleza diversa, entre las cuales se encuentran "los desastres naturales y los de origen humano, el VIH/SIDA y otras enfermedades, otros riesgos a la salud y el deterioro del medio ambiente" (OEA 2003), por ello, en contexto de pandemia, los Estados deben tomar las medidas necesarias para mitigar sus efectos, apoyándose en la cooperación bilateral y multilateral con otros países.

Para poder adoptar esta nueva mirada y enfrentar la seguridad de los Estados en relación a la preparación frente a una emergencia, se debe recurrir a una mirada más amplia, con un enfoque desde la salud pública, y bajo este marco de trabajo se encuentran las Funciones Esenciales de la Salud Pública (FESP) que son un conjunto de medidas que propuso la Organización Mundial de la Salud (OMS) y la Organización Panamericana de la Salud (OPS) para fortalecer la función rectora de la autoridad sanitaria de los Estados en sus distintos niveles de acción e intervención. (Muñoz, et al. 2000; Artaza, 2017)

Específicamente, la onceava FESP se enfoca en la reducción del impacto de emergencias y desastres en salud, función en la cual el sistema de salud debe contar con una Planificación y ejecución de acciones de prevención, mitigación, preparación, respuesta 
y rehabilitación temprana relacionadas con la salud pública. Además, plantea en la misma lógica, debe trabajarse desde un enfoque de múltiple daños y posibilidades de emergencias, teniendo en consideración el liderazgo del sector sanitario con una amplia colaboración multisectorial, (Muñoz, et al. 2000) análisis muy parecido al que nos propone la OPS (2020) a través del documento "Lista de verificación para gestionar los riesgos y los efectos de COVID-2019"; que es una herramienta para ayudar a las autoridades nacionales a desarrollar o revisar los planes de preparación y respuesta ante una pandemia para COVID-19.

En dicho documento, se da énfasis a estrategias dirigidas a la población más vulnerable ya que son quienes se ven más afectados en una pandemia, siendo esta población: a. los enfermos y sus familias, b. las personas con condiciones médicas preexistentes físicas y/o mentales y c. los trabajadores de la salud, especialmente enfermeras y médicos que trabajan directamente con pacientes y en cuarentena. (Ramírez, et al. 2020)

Por todo lo anteriormente descrito, resulta no sólo necesario bajo el contexto actual, sino como aprendizaje futuro, analizar la preparación de los estados frente a un contexto de pandemia; para este estudio en específico, el objetivo fue analizar la preparación para la emergencia del Covid-19 en los países de Argentina, Brasil y México.

\section{ESTRATEGIAS METODOLÓGICAS O MATERIALES Y MÉTODOS}

La metodología utilizada fue cualitativa, descriptiva de análisis documental sobre los países de Argentina, Brasil y México. Para el análisis se utilizó el documento "Lista de verificación para gestionar los riesgos y los efectos de COVID-19" de la Organización Panamericana de la Salud 2020 a través de las variables Planificación de la respuesta, coordinación, recursos, Cuestiones jurídicas y normativas, y Comunicación de riesgos y participación comunitaria.

Los países que conforman la muestra son Argentina, Brasil y México, estos países fueron seleccionados pues concentran la mayor cantidad de población en Latinoamérica, asociado, además, a la diversidad aplicada por estos países en cuanto a las medidas adoptadas para enfrentar la pandemia. (Giraudy, Niedzwiecki, \& Pribble, 2020) Los documentos utilizados para la revisión documental fueron publicaciones de revistas indexadas, entrevistas a actores claves en los diarios y documentación oficial de los gobiernos revisados. Las variables analizadas para dar cuenta de la preparación de una emergencia son: 
a) Planificación de la respuesta: para abordar esta variable se buscaron las subvariables de contexto y alerta, detección, evaluación y clasificación rápida de riesgos.

b) Coordinación: se trabajó en función de la identificación de la coordinación multisectorial entre ministerios de gobierno, autoridades competentes, organizaciones no gubernamentales y agentes no estatales. Junto con aplicar y fortalecer mecanismos de coordinación y comunicación del sector sanitario con asociados en las actividades de preparación, respuesta y recuperación relativas a las pandemias.

c) Recursos: se focalizo la búsqueda del factor humanos para ejecutar, gestionar y coordinar las actividades de respuesta a una pandemia.

d) Cuestiones jurídicas y normativas: se buscó identificar y evaluar la base jurídica de todas las medidas de salud pública que se pudieran proponer durante la respuesta a una pandemia. Además de identificar el asesoramiento de comités de ética sobre actividades de preparación y respuesta para pandemias, en coordinación con las estructuras nacionales pertinentes.

e) Comunicación de riesgos y participación comunitaria: se trabajó en describir el aseguramiento de la disponibilidad de estructuras formales y procedimientos acordados para gestionar la comunicación de riesgos y la participación comunitaria, así como desarrollar mecanismos, como procedimientos de autorización, aseguramiento de la coordinación y la coherencia de las medidas, los mensajes y la participación comunitaria, a nivel nacional, regional y local.

\section{RESULTADOS Y DISCUSIÓN}

A continuación, se presenta el análisis de Argentina, Brasil y México en relación a la preparación de la emergencia según la OPS a través de las cinco variables descritas anteriormente. Estas serán expuestas por país, para luego realizar un resumen esquematizado comparativo de dichos países en cuanto a su abordaje de la pandemia Covid-19 para la reducción del impacto de emergencias y desastres en salud.

\subsection{Argentina}

\subsubsection{Planificación de la respuesta}

En el caso de Argentina, las primeras acciones en el ámbito de la planificación comienzan a fines de enero de 2020, en atención a lo señalado por la OMS, quienes afirman que el nuevo brote de coronavirus es una emergencia de salud pública de importancia internacional (ESPII). Ante este escenario, Argentina como Estado adherente a la OMS 
se compromete a delinear e implementar las acciones recomendadas por este organismo internacional para el control de esta pandemia (Ministerio de Salud Argentina, 2020).

Entre los objetivos a cumplir en la fase de planificación general para evitar la propagación del virus, Argentina definió los siguientes lineamientos, planteados por la OPS: “detectar oportunamente a las personas que padecen COVID-19 y pueden ingresar al país; minimizar la diseminación de la enfermedad, la morbilidad y la mortalidad; asistir en forma adecuada a las personas enfermas; monitorear e investigar brotes que puedan ocurrir; identificar y caracterizar la naturaleza del virus y la gravedad clínica de la enfermedad, recomendar estrategias de manejo específicas de enfermedades respiratorias, contribuir a la recuperación rápida y segura de individuos" (Ministerio de Salud Argentina, 2020, p2).

Se destaca de las acciones adoptadas, la decisión de implementar una planificación consistente en dos etapas: contención y mitigación. Entre los objetivos de la contención se puede destacar la preparación y fortalecimiento del sistema de salud, velar por la detección temprana, minimizar la propagación de la enfermedad y con especial énfasis la entrega de información oportuna, basada en la evidencia científica, de forma responsable para lograr la confianza de la comunidad (Ministerio de Salud Argentina, 2020). En tanto, la fase de mitigación considera una priorización de las atenciones en salud enfocadas en Covid-19, vigilancia epidemiológica, monitoreo de las intervenciones terapéuticas y evaluación de la respuesta del sistema (Ministerio de Salud Argentina, 2020).

En la fase de preparación de la red asistencial, se destaca las acciones necesarias en los hospitales para la reconversión de salas aisladas, establecer los hospitales de referencia para derivación ante casos graves, establecimiento de protocolos de traslados de pacientes graves, canales de comunicación permanente con comités de infecciones para evaluar las medidas de protección adecuadas y asegurar productos de protección personal para los funcionarios de salud. (Ministerio de Salud Argentina, 2020)

Aunque se aprecia un esfuerzo sustancial en el reforzamiento de los equipos de salud, en las tecnologías necesarias y en el propio programa de salud; el desarrollo de la pandemia ha obligado a repensar el perfil de los profesionales de salud de Argentina. (Silberman, et al, 2020). 


\subsubsection{Coordinación interinstitucional}

Otro de los componentes relevantes de evaluar a partir de las recomendaciones de la OPS es la coordinación entre los distintos niveles gubernamentales, agentes no estatales y otros actores relevantes en las acciones frente a la pandemia.

La coordinación interinstitucional se organiza en dos frentes. Uno interno, el cual está circunscrito en la fase de contención, teniendo por objetivo convocar al trabajo conjunto de los actores de nivel ministerial y del sistema sanitario a nivel nacional para ejecutar acciones operativas de preparación y respuesta frente al covid-19. Entre los actores que están convocados a responder a estos objetivos se encuentran, además de las autoridades ministeriales, la Dirección Nacional de Epidemiología e Información Estratégica, Dirección Nacional de Control de Enfermedades Transmisibles, Dirección Nacional de Emergencias Sanitarias, Dirección Nacional de Sanidad y Fronteras, además de sociedades científicas y equipos de comunicaciones del Ministerio de Salud (Ministerio de Salud Argentina, 2020)

En el frente externo, la coordinación interinstitucional bajo la conducción del Ministerio de Salud deberá "conformar red intersectorial con la participación de los sectores de la salud, la educación, el transporte, el turismo, el trabajo, el ambiente, el desarrollo social y todos los demás sectores con participación directa o indirecta sobre actividades dirigidas al cumplimiento de los objetivos establecidos” (Ministerio de Salud Argentina, 2020, p.3)

\subsubsection{Recursos}

Los trabajadores de la salud son aquellas personas encargadas de ejecutar, gestionar y coordinar las actividades de respuesta a la pandemia conforme a las orientaciones dadas por la autoridad sanitaria. Lo cierto es que ninguna planificación ni coordinación será posible si no hay personas capacitadas, comprometidas y motivadas a colaborar en la implementación de lo consignado en directivas, resoluciones decretos de la autoridad sanitaria.

En este ámbito, la opinión de los expertos no presenta una buena evaluación, ya que según la visión de Maureen Birmingham, funcionaria de la OPS/OMS en Argentina "la pandemia por COVID-19 desnudó los desafíos que enfrentan los países en torno a los recursos humanos para la salud, tanto en lo que refiere a los trabajadores de los servicios sanitarios, como los dedicados a la búsqueda activa y aislamiento de cada caso y al rastreo de cada contacto y su cuarentena, o a los microbiólogos trabajando las 24 horas para 
asegurar un diagnóstico oportuno" (OPS Argentina, 2020), se profundiza la crítica enfatizando que los efectos que existen en las capacidades en salud se deben a la falta de inversión arrastrada por muchos años en funciones clave para poder enfrentar una pandemia.

En torno a este punto, el factor crítico de éxito para enfrentar una pandemia ha quedado en evidencia en la mayoría de los países, ya que pese a contar, en algunos casos, con una buena red asistencial y estar preparados para enfrentar una pandemia, la capacitación no ha sido suficiente, no solo por el conocimiento para poder enfrentarla, sino porque se trata de una crisis tan grave y profunda, que los recursos humanos deben ser aumentados y mejorados para enfrentar en con mayor fuerza una pandemia como esta.

\subsubsection{Cuestiones jurídicas y normativas}

Otro aspecto relevante en las recomendaciones de la OPS guarda relación con las cuestiones jurídicas y normativas, entre las cuales se encuentra evaluar una base jurídica de las medidas de salud pública que se pudieran proponer durante la fase de respuesta a una pandemia, además de otras iniciativas relevantes como el establecimiento de comités de ética para que cumplan funciones de asesoría en las actividades de preparación y respuesta a la pandemia.

En cuanto a este último, no hay evidencia de que estas recomendaciones de la OPS estén consideradas en las actividades de planificación para la preparación y respuesta a la pandemia por Covid-19. En cuanto a la base jurídica de las medidas de salud pública la más mediática de todas ha sido la dictación de cuarentenas obligatorias para promover el aislamiento y de esta forma reducir la propagación masiva del virus. Obviamente, dentro de este ordenamiento legal se excluyen los servicios esenciales para el buen funcionamiento del país, lo cual es coherente con lo establecido en las recomendaciones de la OPS, además que el fin no perjudica a las personas, sino que las protege, por lo tanto, la legislación a la restricción de movimiento "se encuentra dentro de límites de proporcionalidad y razonabilidad que hallan su sostén en la Constitución nacional" (Pulvirenti , 2020)

\subsubsection{Comunicación de riesgos y participación comunitaria}

En la planificación establecida por la autoridad sanitaria se considera al componente comunicacional como un eslabón más en la cadena de trabajo en respuesta a la pandemia por Covid-19. En este sentido, la comunicación está considerado como un pilar prioritario 
de la primera fase de contención, teniendo como propósito "Proporcionar al público información actualizada, coherente y precisa sobre el estado de la enfermedad alrededor del mundo y de la situación en Argentina para divulgar el conocimiento necesario para la toma de decisión en cuanto a los cuidados adecuados para evitar la adquisición de la enfermedad" (Ministerio de Salud Argentina 2020), destacando que el objetivo central de las acciones comunicacionales es la creación de confianza pública en la respuesta de los servicios de salud ante el Covid-19.

Entre las acciones determinadas en la fase de planificación, la comunicación de riesgos considera el establecimiento de vocerías, contactos con actores estratégicos para la difusión de información, actualización de plataformas web y manejo de redes sociales. También destaca la intensiva comunicación de recomendaciones sobre los alcances del coronavirus en distintos sectores, rubros y segmentos de la población, como puertos y aeropuertos, escuelas, lugares de aglomeración masiva, empresas y servicios públicos, entre otros (Ministerio de Salud Argentina 2020).

\subsection{Brasil}

\subsubsection{Planificación de la respuesta}

En Brasil, tras un discurso de corte fascista y lleno de una brutal ignorancia, se menospreció la letalidad del virus desde los inicios del mes de marzo 2020, convirtiéndose en un obstáculo para la implementación de medidas de aislamiento y distanciamiento social. Esta postura agravó sobremanera la crisis de poder y la propia grieta que ya se perfilaba en el gabinete de gobierno. En momentos de aunar fuerzas para el combate y enfrentamiento, la crisis política impidió tomar medidas certeras y destinar recursos financieros para enfrentar la situación. (Molina y Mejías, 2020)

Aunque algunas medidas de distanciamiento físico y aislamiento social han sido determinadas por los gobernadores de los estados desde la segunda semana de marzo, la negación de la epidemia por el presidente Bolsonaro, que la llamó 'gripecita' y salió a las calles abrazando y besando personas, minó el cumplimiento de las estrategias de control epidemiológico. Así, desde finales de abril 2020, por presión de sectores económicos e intereses políticos electorales, algunos estados retomaron parte de las actividades económicas suspendidas. (Giovanella et al. 2021, p. 4)

Según el informe de la Organización Panamericana de la Salud OPS, Brasil Colaboró con el Ministerio de Salud para adaptar y poner en práctica las estrategias y directrices de 
vigilancia y análisis de laboratorio. Compartió herramientas analíticas y proporcionó proyecciones para respaldar la toma de decisiones del Gobierno. Ayudó a 27 estados federales a incrementar su plantilla de técnicos de vigilancia y laboratorio y se coordinó con el Ministerio de Salud, el Consejo Nacional de Secretarios de Salud (CONASS) y el Consejo Nacional de Secretarías Municipales de Salud (CONASEMS) para fortalecer las capacidades de vigilancia en los estados y los municipios ante agentes patógenos emergentes y reemergentes. Colaboró con la Secretaría Especial de Salud Indígena para fortalecer la vigilancia y el seguimiento de casos en 100 tierras indígenas. Capacitó a funcionarios del Ministerio de Salud sobre el uso de Go.Data para la localización de contactos. Prestó asistencia al gobierno de Manaus y al estado de Amazonas para fortalecer sus capacidades de laboratorio y de vigilancia ampliando las plantillas laborales (23 enfermeros, 2 enfermeros de biotecnología, 4 farmacéuticos, 3 biólogos y 6 técnicos de enfermería). (OPS 2020, p. 45)

\subsubsection{Coordinación}

En Brasil, las acciones gubernamentales han sido erráticas y desorganizadas, con incapacidad para planificar respuestas públicas coordinadas. En gran medida, los Estados y el Distrito Federal ya en fines de marzo, habían puesto en marcha medidas para limitar el movimiento y la aglomeración de personas en mayor o menor medida. Sin embargo, el Gobierno Federal, al minimizar la importancia de distanciamiento social y oponerse públicamente a las medidas adoptadas por los estados y municipios, socavó la adhesión de la población a ella. (Aquino et al., 2020)

Fortaleció la preparación y la capacidad de respuesta del Brasil ofreciendo aportes técnicos a sus planes y protocolos de urgencia y compartiendo experiencias internacionales. Ayudó al ejecutivo y a la judicatura del país a elaborar protocolos de reducción de la transmisión de COVID-19 entre las poblaciones indígenas y privadas de libertad, según los análisis epidemiológicos y de riesgos de la OPS. Facilitó la coordinación de la respuesta a la emergencia de la COVID-19 entre los tres niveles administrativos del Brasil (federal, estatal y municipal), dentro del Sistema Único de Salud (SUS). Tradujo al portugués las publicaciones y herramientas de la OMS y la OPS sobre COVID-19. (OPS 2020, p. 45)

Colaboró con las autoridades nacionales para que siguieran ofreciéndose, con garantías de seguridad, los servicios de salud esenciales distintos de la COVID-19, como el 
programa de vacunación y el tratamiento de las enfermedades no transmisibles. (OPS 2020, p. 45)

\subsubsection{Recursos}

Prestó apoyo al Ministerio de Salud para elaborar, actualizar y poner en práctica guías de manejo clínico, vigilancia, salud mental y medidas no farmacológicas. Además, evaluó la preparación de los hospitales y trabajó con el Ministerio de Salud para capacitar a más profesionales de salud, ampliando el personal del Sistema Único de Salud (OPS 2020, p. 45). La capacitación en la primera etapa mostró $98,4 \%$ de cumplimiento. Se destacan los directivos con el $100 \%$ de cumplimiento seguido de los licenciados en enfermería con el 99,9 \%. (Núñez, et al, 2020). De acuerdo a lo anterior, realizó actividades de establecimiento de la capacidad para técnicos de laboratorio a fin de mejorar las pruebas y el diagnóstico de la COVID-19. Con esto, aportó kits para pruebas, kits de extracción y otros suministros a los laboratorios nacionales de referencia. Proporcionó a las autoridades locales especificaciones relativas a los EPP, respiradores mecánicos y otros equipamientos. Colaboró con los homólogos nacionales para establecer y aplicar estrategias de gestión de cadáveres en el contexto de la COVID-19. (OPS 2020, p. 45)

Datos extraídos en 2020 de la base de datos del Ministerio de Salud de Brasil, informó un total de 435, 258 camas disponibles en el país. Sobre la base de la población proyectada para el año 2020, esto equivale a unas 22 camas por cada 10.000 habitantes. (Coelho 2020, p. 6)

En la búsqueda para garantizar más dosis para la población brasileña, se firmaron tres acuerdos de transferencia de tecnología en el país. En este contexto, se espera que, en el primer semestre de 2021, tras la aprobación del uso de las vacunas por parte de las agencias reguladoras (en Brasil, la Agencia Nacional de Vigilancia Sanitaria - ANVISA), el país ya comience a recibir las primeras dosis para que sean utilizadas en el territorio nacional. Este será otro gran desafío, puesto que, a pesar de toda la aceleración en el desarrollo observado hasta el momento, todavía existen muchas lagunas de conocimiento, imponiendo una enorme dificultad para organizar el plan de vacunación. (Domíngues 2020, p. 2)

\subsubsection{Cuestiones jurídicas y normativas}

La ley número 13.979, de 6 de febrero de 2020, establece las medidas que podrán adoptarse para hacer frente a la emergencia de salud pública de importancia internacional 
derivada del coronavirus responsable del brote de 2019. Garantiza a las personas afectadas por las medidas previstas el derecho a ser informado permanentemente sobre su estado de salud y asistencia a la familia de acuerdo con la normativa, el derecho a recibir tratamiento gratuito y el pleno respeto a la dignidad, los derechos humanos y las libertades fundamentales de las personas, de acuerdo con el artículo 3 del Reglamento Sanitario Internacional. Considera el período de ausencia resultante de las medidas previstas como ausencia justificada del servicio público o de la actividad laboral privada. Establece la forma y condiciones en que se dispondrán las medidas. (Diario Oficial, 2020, p.14).

Si bien la Ley $\mathrm{N}^{\circ} 13.979$ / 2020, que prevé medidas para combatir el COVID-19 en el país, está vigente desde el 7 de febrero 2020, es decir, desde antes del inicio oficial de la epidemia, el presidente Jair Bolsonaro ha minimizado su importancia, manteniéndose como uno de los pocos líderes mundiales que se niega a reconocer la amenaza que constituye. (Aquino et al., 2020)

El 6 de abril de 2020 se constituyó la Brigada Dr. Alfredo Méndez Aguirre (eminente médico cienfueguero) ante la presencia de las autoridades de la provincia, compuesta por 21 profesores como expertos encargados de asesorar a los municipios y policlínicos en toda la actividad integral y metodológica de enfrentamiento a la epidemia. (Núñez, et al. 2020)

\subsubsection{Comunicación de riesgos y participación comunitaria}

Se formó a líderes comunitarios y de migrantes para que promoviesen las medidas de prevención de la COVID-19. Elaboró y diseminó materiales de comunicación de riesgos diseñados para poblaciones migrantes (en particular en Boa Vista y Roraima) y residentes en albergues, entre otras. Con ayuda del UNICEF, diseñó cursos virtuales para capacitar a los venezolanos residentes en el Brasil a fin de ofrecer atención a la salud mental y apoyo psicosocial a los adolescentes y niños migrantes. (OPS 2020, p. 45)

\subsection{México}

\subsubsection{Planificación de la respuesta}

México, cuenta con un marco constitucional fortalecido que permite interactuar a los tres poderes de la Unión en caso de declararse un Estado de Emergencia (Díaz,2020, p.173), En torno a la respuesta del Índice de Rigor tomado por el gobierno mexicano se encuentra 
entre las más bajas, sin cuarentenas y que presenta una mayor cantidad de población. (Acosta, 2020, p.6)

México tomo una opción de convivir con el virus y sus consecuencias, retomando las formas de convivencia con los virus que implicaba la normalidad de aceptar altas tasas de mortalidad por enfermedades infectocontagiosas, hasta que lográramos el efecto colectivo de inmunidad en forma "natural". Obteniendo como resultado que el $75 \%$ de los muertos por coronavirus en México corresponda a los sectores de escasos recursos económicos. (Menéndez, 2020)

\subsubsection{Coordinación}

México presenta mecanismos suficientes de coordinación y comunicación que fortalecen la toma de decisiones de manera balanceada ante un escenario de emergencia sanitaria, como lo son el Consejo de Salubridad General y la Secretaría de Salud (Díaz 2020, p.173) Se establecieron medidas de control inmediatas una vez se dio la confirmación del virus, es así como la Dirección General de Epidemiología emitió un aviso preventivo de viajes a China, y luego publicó el lineamiento estandarizado para la vigilancia epidemiológica y por laboratorio de enfermedad por Covid-19, (Aragón-Nogales, Vargas-Almanza, Miranda-Novales, 2019)

A pesar de ello el entorno político y financiero mexicano, lograron minar la capacidad de los tomadores de decisiones para implementar una estrategia de contingencia y de mitigación única y uniforme a nivel nacional, esto a través de la falta de trabajo en equipo y trabajo paralelo entre los grupos de la sociedad, la desinformación y los intereses de los grupos políticos y de la iniciativa privada, han (Díaz, 2020, p.173)

\subsubsection{Recursos}

$\mathrm{Al}$ revisar los recursos con que fue enfrentada la pandemia en México nos encontramos con un panorama desalentador antes del inicio de esta crisis socio sanitarias, entre ellas se encuentran, las deficiencias sanitarias como la falta de infraestructura tecnológica en el área de diagnóstico, la falta de recursos humanos especializados, y el desmantelamiento del Sistema de Seguro de Salud Universal (Díaz, 2020, p.173 ) que se vieron acrecentadas por las tardías medidas del gobierno, como la muerte temprana de 212 enfermeras, que corresponde a un 25\% de las muertes a nivel mundial. (Menéndez, 2020) Esto queda claramente reflejado por la inversión en salud en los últimos años, 2019 y 2020 que 
representan el $2,5 \%$ y el $2,7 \%$ del PIB, estando casi un $4 \%$ bajo lo recomendado por la OMS (Menéndez, 2020; Escudero, et al, 2020)

Esto desemboco en que las tasas de incidencias acumuladas fueran entendidas como intermedias en comparación con el resto de los países de la región, pero se reflejaban en altas tasas de letalidad junto con bajos niveles de testeos (Acosta, 2020, p.6)

En el mismo sentido Escudero, et al, (2020) prevén que, si la curva de contagio se mantiene ascendente y los casos no logran ser contenidos, el sistema de salud se verá colapsado, produciendo altos costos presupuestales acompañados de grandes carencias en, equipos, medicamentos e infraestructura.

\subsubsection{Cuestiones jurídicas y normativas}

En el segundo mes de la pandemia el Consejo de Salubridad General publicó una Guía Bioética, donde se explica que al momento de que los insumos falten se "se deberá recurrir al principio de vida completada, es decir que pacientes más jóvenes han de recibir atención de cuidados intensivos sobre pacientes de mayor edad (Menéndez, 2020)

En este sentido queda claro tal como dijo Cossio que los órdenes jurídicos han tenido mecanismos para tratar de preservar un bien común; la colectividad. En este sentido el mayor recurso que tiene la Constitución Política de los Estados Unidos Mexicanos para enfrentar un momento extraordinario como esta crisis es la suspensión de derechos y garantías que no se ha utilizado. La segunda medida son los organismos del Consejo de Salubridad General y la Secretaría de Salud, donde el primero puede determinar y tomar cierto tipo de acciones, y complementarlas con la acción de la Secretaría. Como declarar la atención del Covid-19 prioritaria. (Rendón 2020)

\subsubsection{Comunicación de riesgos y participación comunitaria}

La comunicación del riesgo, debe enfocarse en la sociedad civil y los medios de comunicación no de manera exclusiva en estos últimos, para de esta manera abordar planes de mitigación en las distintas fases de la contingencia. Situación que en este caso no ocurrió, dando pie a respuestas desfasadas entre los diferentes niveles de gobierno y sectores de la sociedad, y la vigilancia epidemiológica. (Díaz 2020, 174)

Al respecto, la información en México coloco el énfasis comunicacional en las consecuencias económicas y ocupacionales de la pandemia. A pesar de que los medios de comunicación entregaban de manera diaria informaban sobre el número de contagios, recuperados y muertos, además de generalidades del virus, el fuerte de la comunicación 
se posicionaba en la crisis económica, los millones de desocupados que genera, y a cómo afrontar la crisis. Llegando a representar el 97\% de las comunicaciones masivas sobre temas económicos (Menéndez, 2020)

\section{CONCLUSIÓN O CONSIDERACIONES FINALES}

De la variable Planificación de la respuesta, destaca Argentina, que siguió las medidas entregadas por la OMS, más se evidencia un déficit en lo aplicado en esta primera etapa por Brasil y México, lo que retrasó una respuesta temprana y oportuna, limitando un abordaje y manejo más adecuada de la pandemia.

En la variable Coordinación, se logra apreciar que Argentina aplico un protocolo estándar de trabajo multisectorial, mientras que en Brasil se evidenció una falta de coordinación entre el gobierno federal y los distritos, y en México el poder económico desarticuló cualquier toma de decisión coordinada.

En la variable Recursos, Argentina presenta una reconversión de trabajadores hacia la pesquisa de nuevos casos, en Brasil se capacita al personal en las necesidades requeridas a nivel clínico y administrativo, mientras en México, que venía con un déficit en infraestructura y recursos humanos previo a la pandemia, el Covid-19 desató un verdadero estado de emergencia que se vio reflejado por el alto porcentaje de personal sanitario fallecido, $25 \%$ del estamento enfermero.

Para la variable de Cuestiones jurídicas y normativas, Argentina cumplió a cabalidad las recomendaciones de la OMS, aplicando restricción de las libertades individuales a través de cuarentenas. Brasil por su parte otorgó beneficios y enfoques legales para combatir el Covid-19. Sin embargo, México, teniendo la potestad legal para aplicar diversas medidas en lo jurídico y normativo decidió no realizarlo desde un inicio, retrasando la decisión, aplicando las medidas demasiado tarde.

Finalmente, la variable Comunicación de riesgos y participación comunitaria, fue la con mayor variabilidad de aplicación, mientras que Argentina hizo uso de vocerías y contactos con actores estratégicos para la difusión de información, así como actualización de plataformas web y manejo de redes sociales. Brasil a través del uso de la multiculturalidad, aplicó diversas medidas de acción, entre estas formar a líderes comunitarios, creando cursos virtuales y desarrollando comunicación atingente para la población migrante. México abordó la pandemia desde una perspectiva económica, desarrollando más de un $97 \%$ de noticias con esta mirada. 
De esto, es posible evidenciar que, en aquellos países con mayor concentración de población, existen ciertas coincidencias; México y Brasil adoptaron una mirada conservadora, que se reflejó en el alto número de casos positivo y muertes asociadas, secundario a las escazas medidas de confinamiento. Por otro lado, Argentina, teniendo la mitad del número de habitantes, logró una mayor capacidad de respuesta frente a la pandemia, aun cuando al igual que los otros países, tiene un tipo de Gobierno Federal.

Si bien, existe evidencia de que Argentina cumplió las recomendaciones de la OMS, esto no garantiza la efectividad en evitar la propagación del virus, puesto que estas recomendaciones deben asociarse a otras medidas para que el sistema pueda gestionar en forma eficiente la pandemia; entre ellas está la fortaleza de la red hospitalaria y la conciencia social y de salud pública de las personas y comunidades.

Cabe destacar que la comunicación del riesgo es de suma importancia y puede marcar una gran diferencia en la respuesta ante cualquier tipo de emergencia, en especial cuando esta es de interés de la salud pública, así también la manera en que actúan los gobiernos de manera coordinada, siguiendo o no las indicaciones de los organismos internacionales en salud para tomar medidas tempranas de mitigación, puede marcar la diferencia en la seguridad de las personas y los países.

\section{LISTA DE REFERENCIAS}

Acosta, L. (2020) Capacidad de respuesta frente a la pandemia de COVID-19 en América Latina y el Caribe. Revista Panamericana Salud Publica. 44(e109), 1-8. https://doi.org/10.26633/RPSP.2020.109

Aquino, Estela M. L., Silveira, Ismael Henrique, Pescarini, Julia Moreira, Aquino, Rosana, Souza-Filho, Jaime Almeida de, Rocha, Aline dos Santos, Ferreira, Andrea, Victor, Audêncio, Teixeira, Camila, Machado, Daiane Borges, Paixão, Enny, Alves, Flávia Jôse Oliveira, Pilecco, Flávia, Menezes, Greice, Gabrielli, Ligia, Leite, Luciana, Almeida, Maria da Conceição Chagas de, Ortelan, Naiá, Fernandes, Qeren Hapuk R. Ferreira, Ortiz, Renzo Joel Flores, Palmeira, Raquel Nunes, Pinto Junior, Elzo Pereira, Aragão, Erika, Souza, Luis Eugenio Portela Fernandes de, Barral Netto, Manoel, Teixeira, Maria Glória, Barreto, Mauricio Lima, Ichihara, Maria Yury, \& Lima, Raíza Tourinho dos Reis Silva. (2020). Medidas de distanciamento social no controle da pandemia de COVID-19: potenciais impactos e desafios no Brasil. Ciência \& Saúde Coletiva, 25(Suppl. 1), 
2423-2446. Epub June 05, 2020. https://dx.doi.org/10.1590/141381232020256.1 .10502020

Aragon-Nogales, R., Vargas-Almanza, I. \& Miranda-Novales, M.. (2019) COVID-19 por SARS-CoV-2: la nueva emergencia de salud. Revista. Mexicana de. pediatria, 86, (6), 213-218, dic. 2019. Disponible en http://www.scielo.org.mx/scielo.php?script=sci_arttext\&pid=S0035$00522019000600213 \& \operatorname{lng}=\mathrm{es} \& \mathrm{nrm}=$ iso

Artaza, O. (2017). Funciones esenciales de salud pública: su implementación en Argentina y desafíos hacia salud universal: experiencia federal 1a ed . - Buenos Aires : Organización Panamericana de la Salud - OPS, 2017 https://iris.paho.org/bitstream/handle/10665.2/34026/9789507101274_spa.pdf?s equence $=1 \&$ is Allowed $=\mathrm{y}$

Coelho C, Flávio, Lana M, Raquel, Cruz G, Oswaldo, Villela Daniel, Bastos S Leonardo, Pastore Ana y Piontti , T Davis, Jessica, Vespignani Alessandro, Codeço T, Claudia, Gomes FC, Marcelo (2020), Evaluación del impacto potencial de COVID-19 en Brasil: Movilidad, Morbilidad y la carga sobre el Sistema de Salud. medRxiv; . doi:https://doi.org/10.1101/2020.03.19.20039131

Díaz - Ramírez, J. B. (2020). Cuando la ciencia no alcanza: México ante la pandemia del COVID19. Horizonte Sanitario, 19(2), 173-174. https://doi.org/10.19136/hs.a19n2.3705.

Domingues, Carla Magda Allan Santos (2021). Desafíos para la realización de la campaña de vacunación contra la COVID-19 en Brasil. Cadernos de Saúde Pública, 37(1), e00344620. Epub January 11. https://doi.org/10.1590/0102-311x00344620

Diário Oficial, (2020). Ley Número 13.970/2020, de 02 de julio, Medidas que podrán adoptarse para hacer frente a la emergencia de salud pública de importancia internacional derivada del coronavirus responsable del brote de 2019. Diário Oficial 14, http://www.ioepa.com.br/pages/2020/2020.07.02.DOE.pdf

Escudero, X., Guarner, J., Galindo-Fraga, A., Escudero-Salamanca, M., Alcocer-Gamba, M, \& Río, C. (2020). La pandemia de Coronavirus SARS-CoV-2 (COVID-19): Situación actual e implicaciones para México. Archivos de cardiología de México, 90(Supl. 1), 7-14. Epub 24 de marzo de 2021.https://doi.org/10.24875/acm.m20000064 
Giovanella, Ligia, Vega, Román, Tejerina-Silva, Herland, Acosta-Ramirez, Naydú, Parada-Lezcano, Mario, Ríos, Gilberto, Iturrieta, Daisy, Almeida, Patty Fidelis de, \& Feo, Oscar. (2021). ¿Es la atención primaria de salud integral parte de la respuesta a la pandemia de Covid-19 en Latinoamérica? Trabalho, Educação e Saúde, 19, e00310142. Epub October 19, 2020. https://doi.org/10.1590/19817746-sol00310

Giraudy, A., Niedzwiecki, S., \& Pribble, J. (30 de abril de 2020). How political science explains countries' reactions to COVID-19. America Quarterly. https://www.americasquarterly.org/article/how-political-science-explainscountries-reactions-to-covid-19

Maguiña C., Gastelo, R., \& Tequen, A. (2020). El nuevo Coronavirus y la pandemia del Covid-19. Revista Medica Herediana, 31(2), 125-131. https://dx.doi.org/10.20453/rmh.v31i2.3776

Ministerio de Salud Argentina. (Febrero de 2020). Ministerio de Salud. Recuperado el 11 de 04 de 2021, de https://cutt.ly/vvqN21I

Menéndez, E. 2020. Consecuencias, visibilizaciones y negaciones de una pandemia: los procesos de autoatención. Salud Colectiva v. 16 : https://doi.org/10.18294/sc.2020.3149

Molina, N. \& Mejias, M. (2020). Impacto social de la COVID-19 en Brasil y Ecuador: donde la realidad supera las estadísticas. EDUMECENTRO, 12(3), 279-280. Epub 22 de junio de 2020. Recuperado en 10 de abril de 2021, de http://scielo.sld.cu/scielo.php?script=sci_arttext\&pid=S2077$28742020000300277 \& \operatorname{lng}=$ es\&tlng=es

Muñoz, F., López-Acuña, D., Halverson, P., Guerra, C., Hanna, W., Larrieu, M., Ubilla, S. y Zeballos.J. (2000). Las funciones esenciales de la salud pública: un tema emergente en las reformas del sector de la salud. Revista Panamericana de Salud Pública 8(1/2), 126- 134. https://www.scielosp.org/pdf/rpsp/v8n1-2/3012.pdf

Núñez, A., Fernández, M., González, Y., Gaimetea , C., Rojas R, \& López, T. (2020). Resultados de la capacitación sobre la COVID-19. Universidad de Ciencias Médicas de Cienfuegos, 2020. MediSur, 18(3), 396-403. Epub 02 de junio de 2020. http://scielo.sld.cu/scielo.php?script=sci_arttext\&pid=S1727897X2020000300396\&lng=es\&tlng=es. 
OEA. "Organización de Estados Americanos". 28 de octubre de 2003. https://cutt.ly/Bvq8E3u (último acceso: 12 de abril de 2021)

OPS Argentina. OPS Argentina. 03 de junio de 2020. https://cutt.ly/4vqMftV (último acceso: 11 de abril de 2021)

Organización Panamericana de la Salud. (2020). Lista de verificación para gestionar los riesgos y los efectos de COVID-2019. https://cutt.ly/PlaU360

Organización Panamericana de la Salud OPS. (2020). Respuesta de la Organización Panamericana de la Salud a la COVID-19 en la región de las américas, 45, https://docs.bvsalud.org/biblioref/2020/06/1100377/respuesta-ops-covid-19americas-31-mayo-2020.pdf

Pulvirenti, O. (8 de abril de 2020). Microjuris. Recuperado el 11 de abril de 2021, de https://cutt.ly/hvqMAra

Ramírez-Ortiz J, Castro-Quintero D, Lerma-Córdoba C, Yela-Ceballos F, EscobarCórdoba F. Mental health consequences of the COVID-19 pandemic associated with social isolation. Colombia Journal of Anesthesiology. 2020;48(4):e930. doi: https://doi.org/10.5554/22562087.e930

Rodríguez-Morales, A., Sánchez-Duque, J., Hernández Botero, S., Pérez-Díaz, C., Villamil-Gómez, W., Méndez, C., Verbanaz, S., Cimerman, S., RodriguezEnciso, H., Escalera-Antezana, J., Balbin-Ramon, G. J, Arteaga-Livias, K., Cvetkovic-Vega, A., Orduna, T., Savio-Larrea, E., \& Paniz-Mondolfi, A. (2020). Preparación y control de la enfermedad por coronavirus 2019 (COVID-19) en América Latina. Acta Médica Peruana, 37(1), 3-7. https://dx.doi.org/10.35663/amp.2020.371.909

Rendón, Pedro (2020) Marco jurídico, única forma de ordenar crisis por COVID: Dr. José Ramón Cossío. Diario en línea. https://ibero.mx/prensa/marco-juridico-unicaforma-de-ordenar-crisis-por-covid-dr-jose-ramon-cossio

Silberman, P., Medina, A., Díaz, J., López, E., \& Dursi, C. (2020). Políticas de Planificación y Gestión del Talento Humano del Ministerio de Salud de Argentina durante la Pandemia de Covid-19. Revista Argentina de Salud Pública. (12) Supl COVID-19:e11. 